\title{
Transcortical Sensory Aphasia as a Result of Left Frontal Cortical-Subcortical Infarction
}

\author{
A Case Report
}

\author{
N.K. Sethi ${ }^{\mathrm{b}} \quad$ L. Burke ${ }^{\mathrm{a}} \quad$ J. Torgovnick ${ }^{\mathrm{b}} \quad$ E. Arsura ${ }^{\mathrm{c}}$ \\ Departments of a Speech Pathology, ${ }^{\mathrm{b}}$ Neurology and ${ }^{\mathrm{C}}$ Medicine, Saint Vincent Hospital and Medical Center, \\ New York, N.Y., USA
}

Dear Sir,

Transcortical sensory aphasia (TSA) typically localizes to the left parieto-occipital cortex and usually is due to an infarct in the inferior division of the left middle cerebral artery. We present here a case of a 75-year-old right-handed woman admitted to our hospital with acute onset of speech and language disturbance. When assessed, her spontaneous speech was fluent, and she had no difficulty in speech initiation and in her ability to repeat short sentences (6-7 words). She did have severe difficulty in visual recognition and aural comprehension of words and in her ability to name objects. Computed tomography (CT) and magnetic resonance imaging showed a left frontal cortical-subcortical infarct. The anatomical correlate of TSA is also discussed.

\section{Case Report}

A 75-year-old, right-hand dominant woman presented with an abrupt onset of speech and language disturbance. The medical history was significant for dyslipidemia for which she was on a statin. The social history included complete independence in all activities of daily living. The patient's primary language was English.
She was reportedly studying Spanish as a second language. A full neurological workup and detailed speech-language assessment were conducted.

Neurological examination revealed a conscious alert lady with intact power in all 4 extremities, grade 5/5 (Medical Research Council grade) and down going plantars. There were no sensory deficits, and cortical sensations were intact. Visual fields could not be assessed reliably on account of the patient's auditory comprehension deficits. Detailed language testing was carried out by a certified speech pathologist on day 3 of stroke. As a part of formal language testing with standardized aphasia batteries, 7 language tasks were administered during testing to assess for spontaneous speech, repetition, auditory comprehension, naming, word and paragraph reading and syllable discrimination. Language assessment revealed fluent verbal output in the presence of impaired auditory comprehension and intact repetition. Spontaneous speech was fluent and effortless. Auditory comprehension was impaired, and the patient had significant difficulty with single-step commands like 'touch your nose' or 'close your eyes'. Re- ceptively, this patient evidenced poor object identification and poor body part identification. Significant verbal perseverations and semantic paraphasic and phonological errors were noted. Impaired reading comprehension was present. Repetition was markedly intact for short phrases of up to 7 words each with an accuracy of word and nonword repetition of $>90 \%$. The patient had significant difficulties with naming on command especially proper nouns. There was no alexia, agraphia, finger agnosia or right-left confusion.

Unenhanced CT and magnetic resonance imaging of the head revealed a left frontal cortical-subcortical infarct (fig. 1, $2,3)$. There was no involvement of the thalamic peduncles or nuclei on imaging. Holter monitoring revealed paroxysmal atrial fibrillation, and the stroke etiology was presumed to be cardioembolic. Language therapy targeting expressive and receptive language was initiated 3 days after the onset of aphasia. The therapeutic goals included improving naming and identification of objects associated with activities of daily living. At the time of discharge to a subacute rehabilitation facility (10 days

\section{KARGER}

Fax +41 613061234 E-Mail karger@karger.ch www.karger.com
(C) 2007 S. Karger AG, Basel 0014-3022/07/0571-0052\$23.50/0

Accessible online at: www.karger.com/ene
Nitin K Sethi, MD

Chief Resident, Neurology, Saint Vincent's Hospital and Medical Center 153 West, 11th Street

New York, NY 10011 (USA)

E-Mail sethinitinmd@hotmail.com 


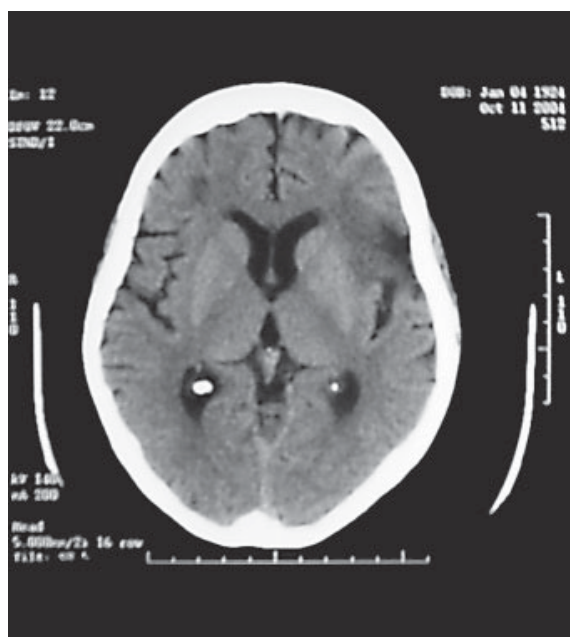

Fig. 1. Head CT scan showing frontal cortical-subcortical infarct.

after her stroke) severe receptive language deficits persisted. The patient was significantly distressed and frustrated by her language handicap.

\section{Discussion}

TSA is characterized by impaired auditory comprehension with intact repetition and fluent speech [1]. It is the sparing of repetition that distinguishes TSA from other receptive aphasias including Wernicke's aphasia and pure word deafness. Repetition requires intact phonological processing, and it is believed that TSA occurs as a result of disruption between left hemisphere phonology and lexical-semantic processing. In other words access to the internal lexicon (memory bank) is lost, so the patient has difficulty in retrieving meaning from words [2]. Thus TSA is a disconnection syndrome. The exact lesional topography of TSA is still a clinicalanatomical puzzle. Classically TSA localizes to the left inferolateral temporo-occipital cortex, though the syndrome has been anatomically correlated with lesions in a variety of posterior brain regions including the anterolateral thalamus and the posterior second and third temporal gyri. It has also been induced transiently by electrical interference during routine cortical function mapping in adult seizure pa-

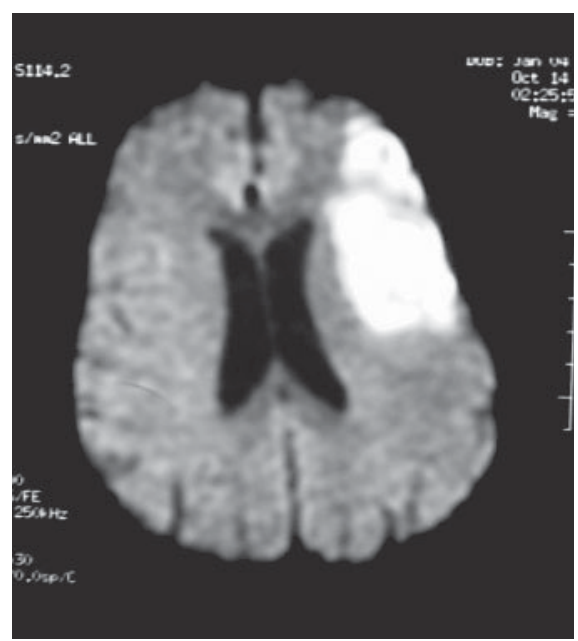

Fig. 2. Brain MRI showing frontal cortical-subcortical infarct.

tients and was mapped to multiple posterior cortical sites, including the posterior superior and middle temporal gyri, in the classical Wernicke area [1]. TSA has been described with a variety of diseases including Alzheimer's disease, Pick's disease as well as traumatic temporal lobe hemorrhages, though it is the stroke cases which are the best candidates for anatomical correlation of this syndrome. Stroke studies have reiterated that infarcts in the inferiormiddle temporal region (Brodman's area 37) as well as posterior temporal-parietaloccipital junction produce TSA. TSA has been described previously due to a left frontal subcortical hemorrhage [3]. Our patient presented with TSA clinically indistinguishable from that caused by damage to the more posterior language areas. The exact mechanism whereby a frontal cortical-subcortical infarct would cause a TSA is not clear, it may occur due to undercutting of some frontal cortical fibers converging on Wernicke's area, or maybe at the time of the infarct our patient suffered a hypoperfusion injury to the more posteriorly placed language areas, though there was no evidence of it on neuroimaging studies which were obtained. The imaging was carried out on the day of presentation (day 0 of stroke), and it might have been too early to visualize the entire ischemic

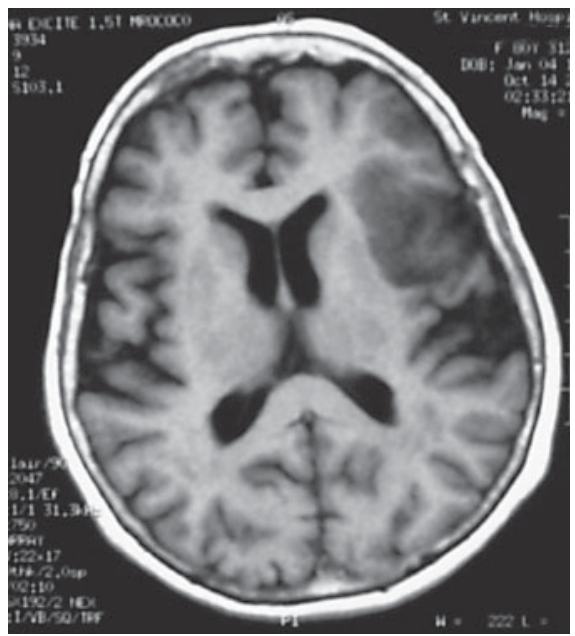

Fig. 3. Brain MRI showing cortical-subcortical infarct.

penumbra. We did not carry out CT perfusion-diffusion imaging, which might have aided in answering the above question. Also cytotoxic edema peaking 2-3 days after the ischemic injury might have contributed to the involvement of more posteriorly situated language areas.

So while we cannot conclusively prove that our patient had a TSA due entirely to a frontal cortical-subcortical lesion rather than involvement of more posteriorly situated language areas, we do want to use this as an excuse to propagate further case reports and studies with newer imaging modalities like positron emission tomography, CT perfusion-diffusion imaging and functional magnetic resonance imaging with the aim of better mapping out language localization in the brain.

\section{References}

1 Boatman D, Gordon B, Hart J, Selnes O, Miglioretti D, Lenz F: Transcortical sensory aphasia: revisited and revised. Brain 2000; 123:1634-1642.

2 Alexander M, Hiltbrunner B, Fischer R: Distributed anatomy of transcortical sensory aphasia. Arch Neurol 1989;46:885-892.

3 Maeshima S, Kuwata T, Masuo O, Yamaga H, Okita R, Ozaki F, Moriwaki H, Roger P: Transcortical sensory aphasia due to a left frontal subcortical hemorrhage. Brain Inj 1999;13:927-933. 